ORIGINAL ARTICLE

\title{
Nuclear $\beta$ catenin as a potential prognostic and diagnostic marker in patients with colorectal cancer from Hong Kong
}

\author{
S C C Wong, E S F Lo, A K C Chan, K C Lee, W L Hsiao
}

J Clin Pathol: Mol Pathol 2003;56:347-352

See end of article for authors' affiliations

Correspondence to: Dr S C Cesar Wong Department of Pathology Queen Elizabeth Hospital, Hong Kong Special Administrative Region, China; cesar01@ netvigator.com

Accepted for publication 5 August 2003

\begin{abstract}
Aims: To study the expression of nuclear $\beta$ catenin in patients with colorectal cancer, colorectal adenoma, and colorectal polyps to elucidate its role in carcinogenesis, and its potential for prognosis and diagnosis. Methods: The expression of nuclear $\beta$ catenin was studied by immunohistochemistry using paraffin wax embedded specimens. Sixty specimens each of colorectal carcinoma, colorectal adenoma, colorectal polyp, and normal colorectal specimens were analysed. The potential for prognosis was assessed by correlating nuclear $\beta$ catenin expression in 60 and 75 patients with colorectal cancer with lymph node metastasis and survival, respectively. The diagnostic capacity was explored by comparing nuclear $\beta$ catenin expression in 60 patients with colorectal cancer with other cytokeratin 20 (CK20) positive adenocarcinomas, namely: 30 colonic mucinous adenocarcinomas, 30 gastric adenocarcinomas, 27 pancreatic adenocarcinomas, and 12 ovarian mucinous adenocarcinomas.

Results: Nuclear $\beta$ catenin expression was highly associated with progression of colorectal tissue from normal epithelial tissue, polyps, adenomas, to carcinomas $(r=0.875 ; \mathrm{p}<0.0001)$. Nineteen patients with colorectal adenoma who subsequently developed colorectal carcinoma had higher nuclear $\beta$ catenin expression than those with colorectal adenomas alone $(p<0.0001)$. Moreover, those patients with colorectal cancer and high nuclear $\beta$ catenin expression had a higher incidence of lymph node metastasis $\left(\chi^{2}=16.99 ; p<0.005\right)$ and shorter overall survival $(p<0.0001)$. Finally, nuclear $\beta$ catenin expression in colorectal adenocarcinomas was significantly higher than in other CK2O positive adenocarcinomas.

Conclusions: Nuclear $\beta$ catenin expression is a potential prognostic factor in patients with colorectal cancer, and together with CK2O, it could be used to identify colorectal carcinoma in the Hong Kong population.
\end{abstract}

B eta catenin was first identified as a protein associated with E-cadherin in maintaining cell-cell interactions. ${ }^{1}$ Normally, $\beta$ catenin is rigorously controlled by upstream regulators in the Wnt signalling cascade, ${ }^{23}$ through degradation by interaction with the adenomatous polyposis coli (APC) protein and glycogen synthase kinase $3 \beta .{ }^{4}$ However, mutations of the $\beta$ catenin, APC, and AXINl genes, or other genes in the Wnt pathway, can lead to translocation of the $\beta$ catenin protein into the nucleus, where it can act as a transcriptional activator, through binding with $\mathrm{T}$ cell factor or the lymphoid enhancer factor family of transcription factors, ${ }^{4}$ turning on target genes such as c-myc and cyclin Dl in colorectal cancer. ${ }^{56}$ Although $\beta$ catenin signalling seems to play an important role in the carcinogenesis of colorectal cancer, the contribution of nuclear $\beta$ catenin to tumour progression is far from clear. In recent years, most of the studies have focused on $\beta$ catenin mutations. ${ }^{78}$ Although some reports indicated that $\beta$ catenin nuclear translocation occurred early in colorectal adenoma, ${ }^{910}$ a recent study suggested that it was involved in the development of intramucosal and invasive colon cancer but not adenoma. ${ }^{11}$

\footnotetext{
"Although $\beta$ catenin signalling seems to play an important role in the carcinogenesis of colorectal cancer, the contribution of nuclear $\beta$ catenin to tumour progression is far from clear"
}

To investigate the involvement of $\beta$ catenin nuclear translocation in colorectal carcinogenesis, immunostaining of $\beta$ catenin was performed on 60 specimens each of colorectal carcinoma, colorectal adenoma, colorectal nonadenomatous polyp (referred to as polyps), and normal colorectal tissue. In addition, 40 specimens from patients with simultaneous occurrence of colorectal carcinomas, colorectal adenomas, and colorectal polyps were also examined. Furthermore, the associations between nuclear $\beta$ catenin expression and lymph node metastasis and survival were investigated in patients with colorectal carcinoma. Another part of the study explored the merit of nuclear $\beta$ catenin as an adjunctive marker for distinguishing colorectal carcinoma from other adenocarcinomas that are positive for cytokeratin 20 (CK20), a common marker for gastrointestinal tumours, ${ }^{12}$ with similar histological phenotypes. Those tumours included colonic mucinous adenocarcinoma, gastric adenocarcinoma, pancreatic adenocarcinoma, and ovarian mucinous adenocarcinoma.

\section{MATERIAL AND METHODS}

\section{Tissue specimens}

For the first part of our study, four groups of paraffin wax embedded specimens were retrieved from the files of the department of pathology, Queen Elizabeth Hospital, Hong Kong. Group 1 specimens (1998-2000) comprised 60 each of colorectal carcinoma, colorectal adenoma, colorectal polyp (hyperplastic, Peutz-Jeghers, juvenile, and inflammatory), and normal colorectal tissue (table 1). Group 2 specimens were obtained from 40 patients with simultaneous occurrence of colorectal carcinomas, colorectal adenomas, and colorectal polyps. The correlation between nuclear $\beta$ catenin and lymph node metastasis was assessed in group 1 patients

Abbreviations: APC, adenomatous polyposis coli; $\mathrm{CK}$, cytokeratin; $\mathrm{IHC}$, immunohistochemistry 


\begin{tabular}{|c|c|}
\hline Variable & Number \\
\hline \multicolumn{2}{|c|}{ Patients with CRC $(n=60)$} \\
\hline \multicolumn{2}{|c|}{ Sex } \\
\hline Male & 39 \\
\hline Female & 21 \\
\hline \multicolumn{2}{|l|}{ Age (years) } \\
\hline Range & $32-91$ \\
\hline Median & 63.8 \\
\hline \multicolumn{2}{|l|}{ TNM tumour stage } \\
\hline $\mathrm{T} 1$ & 0 \\
\hline T2 & 14 \\
\hline T3 & 34 \\
\hline T4 & 12 \\
\hline \multicolumn{2}{|c|}{ TNM lymph node status } \\
\hline No & 20 \\
\hline N1 & 27 \\
\hline N2 & 13 \\
\hline \multicolumn{2}{|c|}{ Degree of differentiation } \\
\hline Well & 0 \\
\hline Moderate & 44 \\
\hline Poor & 16 \\
\hline \multicolumn{2}{|c|}{ Patients with CRA $(n=60)$} \\
\hline \multicolumn{2}{|c|}{ Sex } \\
\hline Male & 35 \\
\hline Female & 25 \\
\hline \multicolumn{2}{|l|}{ Age (years) } \\
\hline Range & $29-77$ \\
\hline Median & 57 \\
\hline \multicolumn{2}{|c|}{ Degree of dysplasia } \\
\hline Mild & 6 \\
\hline Moderate & 40 \\
\hline Severe & 14 \\
\hline \multicolumn{2}{|c|}{ Patients with CRP $(n=60)$} \\
\hline \multicolumn{2}{|l|}{ Sex } \\
\hline Male & 37 \\
\hline Female & 23 \\
\hline \multicolumn{2}{|l|}{ Age (years) } \\
\hline Range & $32-74$ \\
\hline Median & 48 \\
\hline \multicolumn{2}{|l|}{ Type } \\
\hline Hyperplastic & 19 \\
\hline Peutz-Jeghers & 7 \\
\hline Juvenile & 20 \\
\hline Inflammatory & 14 \\
\hline
\end{tabular}

with carcinoma. To evaluate the prognostic significance of nuclear $\beta$ catenin in adenoma, group 3 specimens from 19 patients with colorectal adenoma who subsequently developed colorectal carcinoma were selected (table 2) and compared with group 1 patients with adenoma alone. Group 4 specimens (1994-1997) were from 75 patients with colorectal cancer (table 3 ) in whom the association between nuclear $\beta$ catenin expression and survival was studied. The second part of the study comprised various CK20 positive adenocarcinomas, namely: 30 colonic mucinous adenocarcinomas, 30 gastric adenocarcinomas, 27 pancreatic adenocarcinomas, and 12 ovarian mucinous adenocarcinomas. The nuclear $\beta$ catenin expression of those specimens was compared with that of group 1 colorectal carcinoma specimens.

\section{Antibody}

We used the anti- $\beta$ catenin monoclonal antibody (C19220; Trasduction Laboratories, Lexington, Kentucky, USA) which was specific for the C-terminal of the human $\beta$ catenin protein (amino acids 571-781).

\section{Immunohistochemical staining and evaluation}

Tissue sections ( $4 \mu \mathrm{m}$ thick) were cut and antigen retrieval was achieved by boiling the sections in EDTA buffer, pH 8.0, in a pressure cooker for 2.5 minutes. Staining was performed
Table 2 Clinicopathological data of group 3 patients (patients with colorectal adenoma who subsequently developed colorectal carcinoma; $n=19$ )

\begin{tabular}{ll}
\hline Variable & Number \\
\hline Sex & 12 \\
Male & 7 \\
Female & \\
Age (years) & $34-69$ \\
Range & 54 \\
Median & \\
Degree of dysplasia & 2 \\
Mild & 9 \\
Moderate & 8 \\
Severe & \\
Years before colorectal carcinoma developed & 0 \\
1 & 4 \\
2 & 6 \\
3 & 5 \\
4 & 4 \\
5 & \\
TNM tumour stage & 0 \\
T1 & 5 \\
T2 & 11 \\
T3 & 3 \\
T4 & \\
TNM lymph node status & 6 \\
N0 & 9 \\
N1 & 4 \\
\hline
\end{tabular}

Table 3 Clinicopathological data of group 4 patients (patients with colorectal carcinoma)

\begin{tabular}{|c|c|}
\hline Variable & Number \\
\hline \multicolumn{2}{|c|}{ Patients with IHC scores $>300(n=25)$} \\
\hline \multicolumn{2}{|l|}{ Sex } \\
\hline Male & 16 \\
\hline Female & 9 \\
\hline \multicolumn{2}{|l|}{ Age (years) } \\
\hline Range & $36-84$ \\
\hline Median & 63.6 \\
\hline \multicolumn{2}{|c|}{ TNM tumour stage } \\
\hline $\mathrm{Tl}$ & 3 \\
\hline T2 & 6 \\
\hline $\mathrm{T} 3$ & 11 \\
\hline $\mathrm{T} 4$ & 5 \\
\hline \multicolumn{2}{|c|}{ TNM lymph node status } \\
\hline NO & 6 \\
\hline NI & 16 \\
\hline N2 & 3 \\
\hline \multicolumn{2}{|c|}{ Degree of differentiation } \\
\hline Well & 0 \\
\hline Moderate & 16 \\
\hline Poor & 9 \\
\hline \multicolumn{2}{|c|}{ Patients with IHC scores $<150(n=18)$} \\
\hline \multicolumn{2}{|c|}{ Sex } \\
\hline Male & 13 \\
\hline Female & 5 \\
\hline \multicolumn{2}{|l|}{ Age (years) } \\
\hline Range & $31-75$ \\
\hline Median & 57.9 \\
\hline \multicolumn{2}{|c|}{ TNM tumour stage } \\
\hline $\mathrm{Tl}$ & 3 \\
\hline T2 & 8 \\
\hline T3 & 5 \\
\hline $\mathrm{T} 4$ & 2 \\
\hline \multicolumn{2}{|c|}{ TNM lymph node status } \\
\hline No & 15 \\
\hline NI & 2 \\
\hline N2 & $\overline{1}$ \\
\hline \multicolumn{2}{|c|}{ Degree of differentiation } \\
\hline Well & 0 \\
\hline Moderate & 13 \\
\hline Poor & 5 \\
\hline
\end{tabular}


in a Ventana-ES automated immunostainer (Tucson, Arizona, USA) at $37^{\circ} \mathrm{C}$ using a $1 / 200$ dilution of the anti- $\beta$ catenin antibody. Normal colorectal epithelial tissues, with membranous $\beta$ catenin staining, were used as positive controls, and negative controls were performed by replacing the antibody with Tris buffered saline. Positive signals were evaluated in four fields under light microscopy at a magnification of $\times 600$, by two independent assessors with out knowledge of clinical outcomes, and in the case of disagreement, consensus was reached after thorough discus sion using a multiheaded microscope. The results were averaged and expressed as immunohistochemistry (IHC) scores obtained by multiplying the "percentage of positive cells" by the "staining intensity", as described previously. ${ }^{13}$ Staining intensity was scored as follows: 0, negative; 1 , weak; 2, moderate; 3 , strong; and 4, very strong. The IHC score ranged from 0 to 400.

\section{Statistical methods}

The relations between nuclear $\beta$ catenin IHC scores and pathological stages of colorectal cancer, lymph node metastasis, and survival were studied using Spearman's rank correlation, $\chi^{2}$, and Kaplan-Meier tests, respectively. GraphPad Prism software version 3.0 (GraphPad Software Inc, San Diego, California, USA, 1994-1999) was used for all statistical analyses and $\mathrm{p}<0.05$ was considered significant.

\section{Ethics}

Our study was approved by the local institutional research ethics committee. Permission for obtaining data from hospital records was granted by the department of pathology, Queen Elizabeth Hospital, Hong Kong Special Administrative Region.

\section{RESULTS \\ $\beta$ Catenin expression in adjacent normal colorectal epithelial tissues}

Membranous $\beta$ catenin expression was seen in all adjacent normal colorectal epithelial tissues (fig lD) and was used as an internal positive control.

\section{Pathological stages of colorectal cancer}

Of the group 1 specimens, all 60 colorectal carcinomas, 55 of the 60 colorectal adenomas, and five of the 60 colorectal polyps, which were either Peutz-Jeghers or hyperplastic polyps, had nuclear $\beta$ catenin expression (fig $1 \mathrm{~A}-\mathrm{D}$ ). The staining pattern of the carcinomas varied greatly, with IHC scores ranging from 100 to 400 (median, 289). In addition, all adenoma cases had fewer positive cells and the staining was weaker, with IHC scores ranging from 0 to 240 (median, 55) (fig 1A,B). Normal colorectal tissue did not exhibit nuclear staining, showing a membranous localisation of $\beta$ catenin (fig 1D). Similar results were obtained for group 2 specimens with a simultaneous occurrence of colorectal carcinomas, adenomas, and polyps (fig $1 \mathrm{E}-\mathrm{G}$ ). Nuclear $\beta$ catenin was seen in all 40 carcinomas, 36 of 40 of adenomas, and three polyps. Interestingly, the IHC scores were highly correlated with the pathological stage of colorectal cancer in both groups of specimens (fig 2A, B) (Spearman's rank correlation test: group 1 specimens, $r=0.875, \mathrm{p}<0.0001$; group 2 specimens, $r=0.723, \mathrm{p}<0.0001)$. Moreover, the IHC scores of adenomas with simultaneously occurring carcinomas (median, 167) were significantly higher than those of adenomas alone (median, 55) (Mann Whitney test, $\mathrm{p}<0.0001$ ).

\section{Patients with colorectal adenoma who subsequently} developed colorectal carcinoma

All but one of the adenoma specimens belonging to patients who subsequently developed carcinomas expressed high amounts of nuclear $\beta$ catenin, with IHC scores ranging from
79 to 255 . The median IHC score of all 19 specimens was 165 , which was much higher than that of group 1 adenoma specimens, which had a median of only 55 (Mann Whitney test, $p<0.0001$ ) (fig $2 \mathrm{C}$ ), but was comparable to that of group 2 colorectal adenoma specimens, which had a median of 167.

\section{Lymph node metastasis}

Carcinoma specimens from group 1 with high $(>300)$ and low $(<150)$ IHC scores were studied. The stratification of cases into two groups was empirical because specimens with nuclear $\beta$ catenin IHC scores $>300$ and $<150$ had large differences both in the percentage of positive cells and the intensity of staining. Of the 60 group 1 specimens, 23 of the 27 specimens from the high IHC group, but only one of 12 cases in the low IHC group had lymph node metastases. The $\chi^{2}$ test showed that the association between nuclear $\beta$ catenin expression and lymph node metastasis was highly significant $\left(\chi^{2}=16.99 ; \mathrm{p}<0.005\right)$.

\section{Survival of patients with colorectal cancer}

Twenty five and 18 of the 75 patients with colorectal cancer had nuclear $\beta$ catenin IHC scores $>300$ and $<150$, respectively. All 25 patients with IHC scores $>300$ and only five of the 18 patients with scores $<150$ died. Kaplan-Meier analysis indicated that the survival curves for these two groups of patients were significantly different $(p<0.0001)$, with a median survival of 14 months for patients with IHC scores $>300$ (fig 3 ).

\section{Nuclear $\beta$ catenin in other CK20 positive adenocarcinomas}

Two of 30 colonic mucinous adenocarcinomas, none of the gastric adenocarcinomas, one of the 27 pancreatic adenocarcinomas, and one of the 12 ovarian mucinous adenocarcinomas showed nuclear $\beta$ catenin positivity, which was focally present in only a small proportion of the cells with weak intensity (mean IHC scores of 35) (fig $1 \mathrm{H}-\mathrm{K}$ ). The findings were in contrast to those of the colorectal adenocarcinomas in group 1, which had a median IHC score of 289 (Mann Whitney test, $\mathrm{p}<0.0001$ ).

\section{DISCUSSION}

$\beta$ Catenin is a multifunctional protein that is both an integral component of cell adhesion molecules and a pivotal member of a signal transduction pathway. ${ }^{12}$ The activation of $\beta$ catenin to an oncogenic state is a consequence of the inactivation of the tumour suppressor APC, by direct mutation of the $\beta$ catenin gene, or by the activation of other Wnt signalling molecules. ${ }^{34} \beta$ Catenin is currently thought to be involved in the development of colorectal cancer, ${ }^{2}{ }^{14}$ which is the second leading cause of cancer deaths in Western countries. ${ }^{2-4}$ Because detection and treatment at an early stage can dramatically improve survival, there has been great interest in developing diagnostic and prognostic factors that can help to detect asymptomatic colorectal carcinoma, as well as its precursor, colorectal adenoma, and to predict the outcome of this cancer accurately. ${ }^{15}{ }^{16}$ However, there is no consensus among researchers on the diagnostic and prognostic potential of $\beta$ catenin in patients with colorectal carcinoma, ${ }^{15-17}$ and its expression and possible roles in patients with colorectal adenoma ${ }^{11} 18$ and polyps. ${ }^{1819}$ Our study is the first to investigate its prognostic and diagnostic potential and to clarify its expression in adenomas and polyps extensively by selecting patients with simultaneously occurring carcinomas, adenomas, and polyps. Our results clearly showed that the expression of nuclear $\beta$ catenin increased significantly during the progression from normal to carcinoma stages for both group 1 and 2 specimens. This reaffirms 

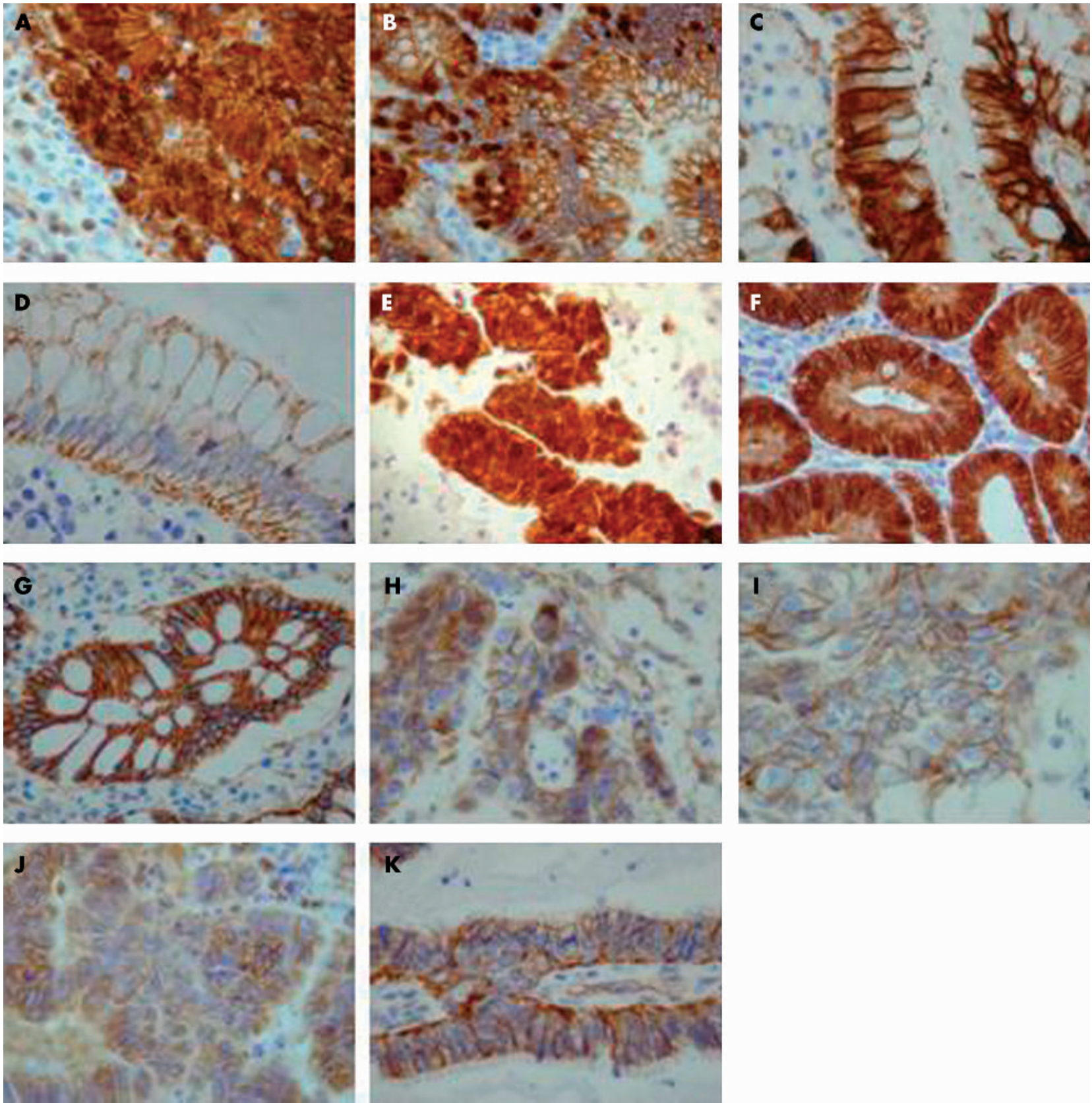

Figure 1 Immunostaining for $\beta$ catenin. (A) Moderately differentiated colon adenocarcinoma; (B) colon adenoma with moderate dysplasia; (C) PeutzJeghers polyp; (D) normal colon epithelium. (E-G) Patient specimen with simultaneously occurring rectal carcinoma, adenoma, and polyp, respectively; $(\mathrm{H}-\mathrm{K})$ colon mucinous adenocarcinoma, stomach adenocarcinoma, pancreas adenocarcinoma, and ovarian mucinous adenocarcinoma, respectively. Original magnification, $\times 600$.

$\beta$ catenin as a key oncogenic factor in colorectal carcinogenesis. ${ }^{14}$ Moreover, for the first time we have shown that the expression of nuclear $\beta$ catenin is significantly higher in adenomas with synchronous carcinomas than in pure adenomas alone, providing evidence that high expression of nuclear $\beta$ catenin is associated with a higher cancer risk. This hypothesis was further explored by studying nuclear $\beta$ catenin expression in 19 patients with adenoma who later developed carcinoma. We found that the expression of nuclear $\beta$ catenin was much higher in those 19 patients. The presence of nuclear $\beta$ catenin in adenomas is in line with the results of Herter et $a l^{18}$ but contrasts with those of Kobayashi et al, ${ }^{11}$ who found no nuclear signals in the adenomas studied. There could be two reasons for this. The first may be the ethnic differences between the two groups studied and the other may be the different antigen retrieval systems used in the two studies. Indeed, we tested out the antigen retrieval protocol used by Kobayashi et al and found that the signals were much weaker than those obtained after pressure cooking. In addition, we found that nuclear $\beta$ catenin was expressed in a small number of Peutz-Jeghers and hyperplastic polyps, not only suggesting that these polyps have a higher malignant potential, which correlates well with the findings of Back et al and Leggett et al, ${ }^{1920}$ but also indicating that deregulation of the $\beta$ catenin pathway occurs early in colorectal carcinogenesis. An in depth investigation of $\beta$ catenin immunostaining in endoscopic colorectal adenoma and polyp biopsies is necessary to assess the risk for those patients of developing 

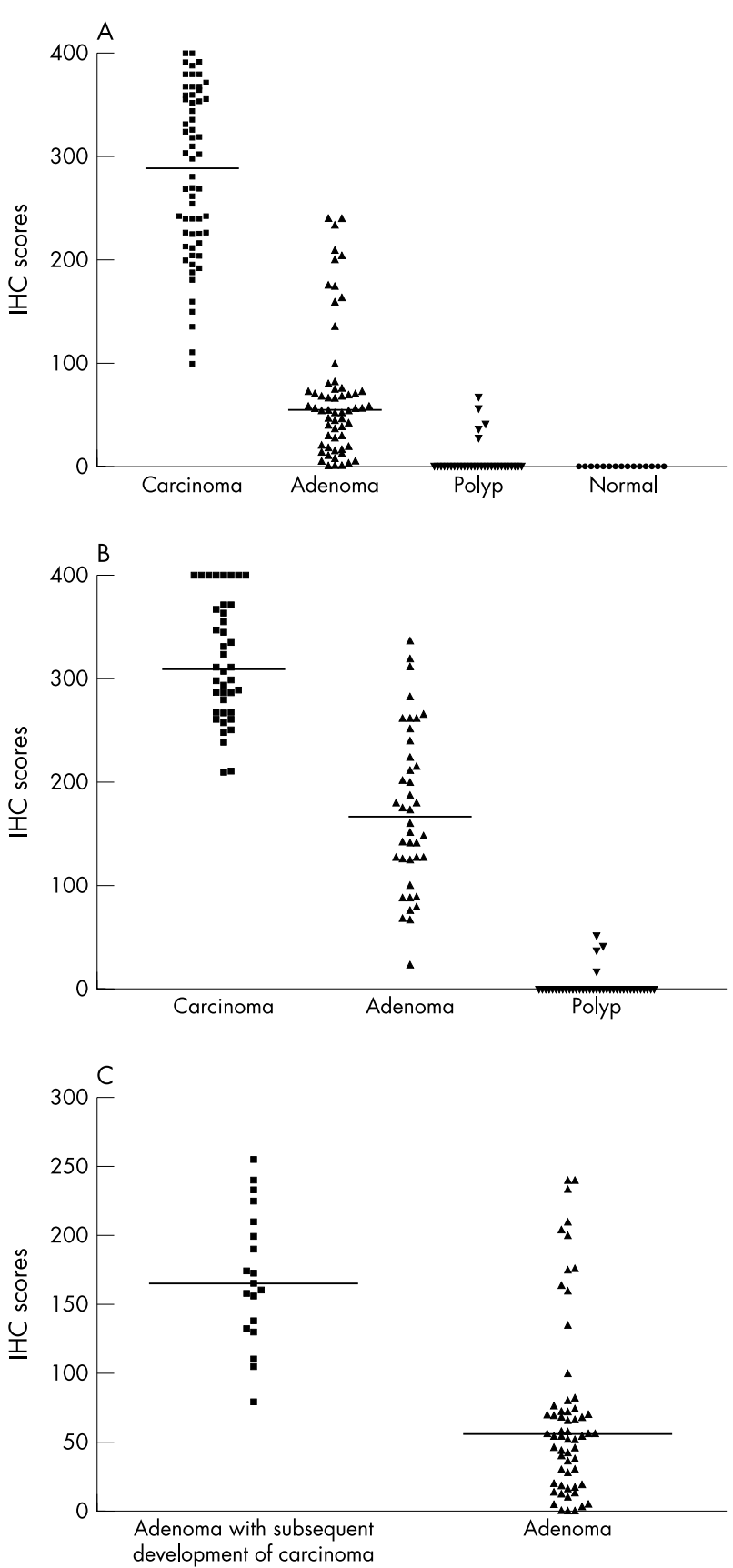

Figure 2 Nuclear $\beta$ catenin immunohistochemistry (IHC) scores. (A) Sixty patient specimens each of colorectal carcinoma, adenoma, polyp, and normal colon tissue; (B) 40 patient specimens each with simultaneous occurring colorectal carcinoma, adenoma, and polyp; (C) 19 patients with adenoma who subsequently developed carcinoma and 60 group 1 patients with adenoma.

colorectal carcinoma in the future. We only identified 19 patients with adenoma who subsequently developed carcinoma, and that sample size was too small to make any conclusions about the size of the cancer risk of nuclear $\beta$ catenin expression in patients with adenoma.

"For the first time we have shown that the expression of nuclear $\beta$ catenin is significantly higher in adenomas with synchronous carcinomas than in pure adenomas alone, providing evidence that high expression of nuclear $\beta$ catenin is associated with a higher cancer risk"

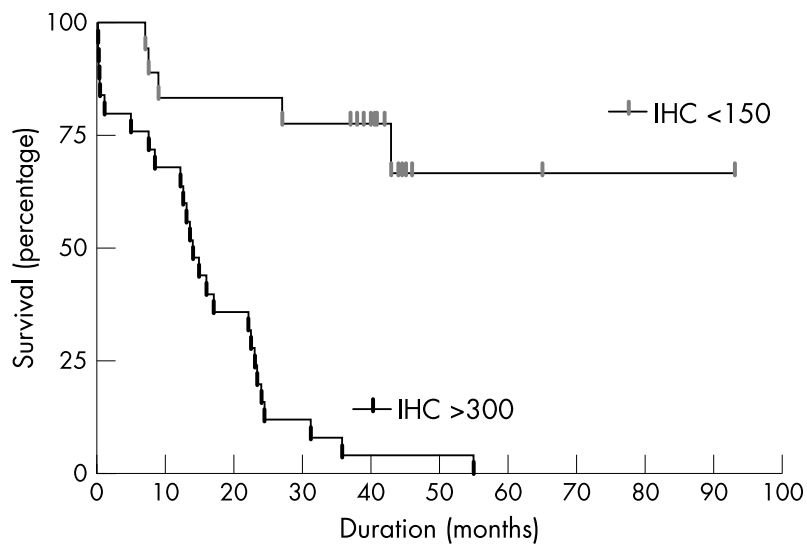

Figure 3 Kaplan-Meier survival curves for patients with colorectal cancer who had nuclear $\beta$ catenin immunohistochemistry (IHC) scores $>300$ and $<150$

Nuclear $\beta$ catenin expression was highly correlated with both lymph node metastasis and patient survival, supporting the suggestion by previous authors ${ }^{15} 16$ that nuclear $\beta$ catenin expression could be used as a powerful prognostic indicator. However, these results differ from those of Gunther et al, ${ }^{17}$ perhaps because different ethnic groups may have different clinical outcomes or because the antigen retrieval and immunohistochemical techniques used in 1998 were less sensitive than those used in recent years.

Finally, because nuclear $\beta$ catenin was expressed in colorectal adenocarcinomas, but not in other CK20 positive adenocarcinomas, staining for nuclear $\beta$ catenin and CK20 could be used to identify colorectal carcinoma. The low nuclear $\beta$ catenin IHC scores found in colorectal mucinous adenocarcinoma implies that this tumour does not exploit the $\beta$ catenin related pathway in carcinogenesis. A larger study is needed to elucidate whether staining for nuclear $\beta$ catenin and CK20 could be used as a novel colorectal cancer marker, but we have already detected 20 nuclear $\beta$ catenin positive metastatic colorectal cancers from various sites (data not shown).

In line with earlier reports, ${ }^{21}{ }^{22}$ heterogeneous expression of $\beta$ catenin was also found in our cohort, perhaps as a result of variations in tumour differentiation. Our results are reliable,

\section{Take home messages}

- Nuclear $\beta$ catenin expression increased significantly with the progression of colorectal tissue from normal epithelial tissue, polyps, adenomas, to carcinomas

- Nuclear $\beta$ catenin expression was higher in patients who subsequently developed colorectal carcinoma than in those with colorectal adenoma alone

- Patients with colorectal cancer and high nuclear $\beta$ catenin expression had a higher incidence of lymph node metastasis and shorter overall survival

- Nuclear $\beta$ catenin expression in colorectal adenocarcinomas was significantly higher than in other cytokeratin 20 positive adenocarcinomas

- Thus, nuclear $\beta$ catenin expression is a potential prognostic factor in patients with colorectal cancer, and together with cytokeratin 20, it could be used to identify colorectal carcinoma in the Hong Kong population 
not only because normal colorectal tissue was used as an internal positive control in each section, and external negative controls were also included in each batch of staining, but also because our evaluation method has been used previously by us, ${ }^{13} 23$ and should be reliable for scoring purposes.

Taken together, the findings of our study will undoubtedly advance our understanding of the role of nuclear $\beta$ catenin in colorectal carcinogenesis and, in addition, suggest that nuclear $\beta$ catenin, despite different outcomes in different ethnic groups, is a potential prognostic and diagnostic marker in patients with colorectal cancer from Hong Kong.

\section{ACKNOWLEDGEMENTS}

This work was funded by a Ho Hung-Chiu Medical Education Foundation Research Grant.

\section{Authors' affiliations}

S C C Wong, E S F Lo, A K C Chan, K C Lee, Department of Pathology, Queen Elizabeth Hospital, Hong Kong Special Administrative Region, China

W L Hsiao, Department of Biology, The Hong Kong University of Science and Technology, Clear Water Bay, Hong Kong Special Administrative Region, China

\section{REFERENCES}

1 Ben-Ze'ev A. The dual role of cytoskeletal anchor proteins in cell adhesion and signal transduction. Ann N Y Acad Sci 1999;886:37-47.

2 Morin PJ. $\beta$-Catenin signaling and cancer. Bioessays 1999:21:1021-30.

3 Willert K, Nusse R. $\beta$-Catenin: a key mediator of Wnt signaling. Curr Opin Genet Dev 1998;8:95-102.

4 Hecht A, Kemler R. Curbing the nuclear activities of $\beta$-catenin. EMBO Rep 2000;1:24-8

5 He TC, Sparks AB, Rago C, et al. Identification of c-MYC as a target of the APC pathway. Science 1998;281:1509-12.

6 Chen RH, Ding WV, McCormick F. Wnt signaling to $\beta$-catenin involves two interactive components. Glycogen synthase kinase- $3 \beta$ inhibition and activation of protein kinase C. J Biol Chem 2000;275:17894-99.

7 Kitaeva MN, Grogan L, Williams JP, et al. Mutations in $\beta$-catenin are uncommon in colorectal cancer occurring in occasional replication errorpositive tumors. Cancer Res 1997;57:4478-81.
8 Iwao K, Nakamori S, Kameyama M, et al. Activation of the $\beta$-catenin gene by interstitial deletions involving exon 3 in primary colorectal carcinomas without adenomatous polyposis coli mutations. Cancer Res 1998;58:1021-26.

9 Inomata M, Ochiai A, Akimoto, et al. Alteration of $\beta$-catenin expression in colonic epithelial cells of familial adenomatous polyposis patients. Cancer Res 1996;56:2213-17.

10 Valizadeh A, Karayiannakis AJ, El-Hariry I, et al. Expression of E-cadherinassociated molecules $(\alpha-\beta$ - and $\gamma$-catenins and $\mathrm{p} 120)$ in colorectal polyps. Am J Pathol 1997;150:1977-84.

11 Kobayashi M, Honma T, Matsuda Y, et al. Nuclear translocation of $\beta$-catenin in colorectal cancer. Br J Cancer 2000;82:1689-93.

12 Lassmann S, Baver M, Soong R, et al. Quantification of CK20 gene and protein expression in colorectal cancer by RT-PCR and immunohistochemistry reveals inter- and intratumour heterogeneity. J Pathol 2002; 198:198-206

13 Wong SC, Lo SF, Lee KC, et al. Expression of frizzled-related protein and Wnt-signalling molecules in invasive breast tumours. J Pathol 2002;196: 145-53.

14 Wong NA, Pignatelli $M$. $\beta$-catenin-a linchpin in colorectal carcinogenesis? Am J Pathol 2002;160:389-401.

15 Hugh TJ, Dillon SA, Taylor BA, et al. Cadherin-catenin expression in primary colorectal cancer: a survival analysis. Br J Cancer 1999;80:1046-51.

16 Cheah PY, Choo PH, Yao J, et al. A survival-stratification model of human colorectal carcinomas with beta-catenin and p27kipl. Cancer 2002:95:2479-86

17 Gunther K, Brabletz T, Kraus C, et al. Predictive value of nuclear beta-catenin expression for the occurrence of distant metastases in rectal cancer. Dis Colon Rectum 1998:41:1256-61.

18 Herter $\mathbf{P}$, Kuhnen $C$, Muller K-M, et al. Intracellular distribution of $\beta$-catenin in colorectal adenomas, carcinomas and Peutz-Jeghers polyps. J Cancer Res Clin Oncol 1999; 125:297-304.

19 Back W, Loff S, Jenne D, et al. Immunolocalisation of $\beta$ catenin in intestinal polyps of Peutz-Jeghers and juvenile polyposis syndromes. J Clin Pathol 1999;52:345-49.

20 Leggett BA, Devereaux B, Biden K, et al. Hyperplastic polyposis: association with colorectal cancer. Am J Surg Pathol 2001;25:177-84.

21 Nakanishi Y, Ochiai A, Akimoto S, et al. Expression of E-cadherin, $\alpha$-catenin, $\beta$-catenin and plakoglobin in esophageal carcinomas and its prognostic significance: immunohistochemical analysis of 96 lesions. Oncology 1997;54:158-65

22 Zheng Z, Pan J, Chu B, et al. Downregulation and abnormal expression of Ecadherin and $\beta$-catenin in nasopharyngeal carcinoma: close association with advanced disease stage and lymph node metastasis. Hum Pathol 1999;30:458-66.

23 Wong SC, Chan KC, Lee KC, et al. Differential expressions of p16/p21/p27 and cyclin DI/D3, and their relationships to cell proliferation, apoptosis and tumour progression in invasive breast ductal carcinoma. J Pathol $2001 ; 194: 35-42$ 